\title{
Fortalezas y debilidades de los inventarios de cementos para su empleo en análisis de ciclo de vida (ACV)
}

\author{
A. AGUADO' 1 , A. JOSA 1 , A. CARDIM ${ }^{2}$ \\ ${ }^{1}$ E.T.S. Ingenieros de Caminos, Canales y Puertos. Barcelona. UPC. 08034 Barcelona (España) \\ ${ }^{2}$ Escuela Politécnica de la Universidad de Pernambuco (Brasil).
}

\begin{abstract}
El análisis del ciclo de vida (ACV) de productos se ha convertido en una herramienta usual de cuantificación de aspectos medioambientales de los mismos. Esta técnica, está bastante extendida en productos industriales estándar, pero no tanto, en productos del sector de la construcción, como es el cemento. Por otro lado, para la realización de un ACV fiable es necesario contar con inventarios, asimismo fiables, lo cual adquiere gran importancia ya que en España no existen de forma sistemática. En este artículo se hace un análisis de los inventarios públicos a los que se ha tenido acceso, correspondientes a diferentes cementos del ámbito europeo, reflejándose las fortalezas y debilidades de los mismos.
\end{abstract}

Palabras clave: análisis de ciclo de vida $(A C V)$, cemento, energía, inventarios.

\section{Strengths and weaknesses of life cycle inventories (LCI) of cement for its use in life assesments (LCA)}

Life-Cycle Assessment (LCA) has become a common and accepted tool for quantifying the environmental impact of products. It is frequently used in some industrial sectors but this is not the case for construction products like cement. Reliable LCA require the use of reliable LifeCycle Inventories (LCI); this is an important point because in Spain reliable LCI are scarce. An analysis of available LCI of different European cements is presented in this paper. This analysis includes the strengths and weaknesses of such inventories.

Key words: Life Cycle Analysis, cement, energy, inventories

\section{INTRODUCCIÓN}

El análisis de ciclo de vida (ACV) es una herramienta de evaluación ambiental de productos, en la que se consideran diferentes impactos ambientales resultantes de las emisiones derivadas de la fabricación de los mismos. En el ámbito internacional esta metodología se ha desarrollado, principalmente en productos industriales, si bien en el sector de la construcción, su extensión es significativamente menor, incluso en ese ámbito. Dentro del sector de la construcción en España, hay que señalar que se está en primeras etapas, lo que requerirá la realización de inventarios propios y no extrapolaciones internacionales, ya que hay que tener presente el carácter local de la construcción. Si se suma ambos aspectos (carácter de iniciación de estos temas en España y la escasa extensión de uso en el sector de la construcción internacional) surge la necesidad de analizar los inventarios existentes, cara a plantear actuaciones futuras, con credibilidad en nuestro ámbito.

En este artículo, consecuencia de la tesis doctoral de A. Cardim [1], se hace una revisión de los inventarios internacionales disponibles en el sector del cemento, entre los que se encuentran los aportados por INTRON [2] y a los existentes en la base de datos del SimaPro [3], correspondientes a países del norte de Europa. En esa revisión se enfatiza tanto en diversos aspectos de las entradas, tales como: empleo de recursos y la contribución de la energía, como en las salidas, especialmente en las siguientes cargas ambientales: dióxido de carbono, óxidos de nitrógeno, dióxido de azufre y material en suspensión en el aire (polvo). Por otro lado, en este análisis se diferencia el tipo de efecto en función del ámbito (local, regional o global), lo que permite una estrategia cara a hacer una política escalonada de toma de decisiones en temas medioambientales.

Estos trabajos se han realizado en el contexto de un proyecto financiado por el Instituto Español del Cemento y sus Aplicaciones (IECA) y pueden servir de base para la preparación de un inventario de los cementos españoles. En la citada tesis doctoral de A. Cardim [1], se hace una propuesta al respecto que las limitaciones de espacio no permiten incorporar en este artículo.

\section{CARACTERÍSTICAS DE LOS INVENTARIOS}

En los estudios de fiabilidad de los inventarios que se requieren, es preciso elegir un proceso simple para no introducir, desde el punto de vista de método científico, más variables que distorsionen el análisis. Por ello, se toma como proceso de referencia, la fabricación de un (1) $\mathrm{kg}$ de cemento, realizando un ACV de esta unidad de análisis, el cual no es objeto de este artículo. Los límites comprenden las fases desde la extracción de las materias primas para la fabricación del clinker hasta la salida de fábrica del producto.

Los inventarios de los que se ha dispuesto en este trabajo se muestran en la tabla 1. Estos corresponden a los aportados por INTRON [2] y por SimaPro [3]. Este último inventario se engloba dentro de la documentación suministrada con el programa SimaPro y corresponden a ejemplos de cementos tipo (I, II, III y IV), dentro de una estructura más general en la que se incluyen materiales muy diversos. Por su parte el inventario correspondiente a INTRON es un inventario específico del sector (CEMBUREAU), si bien obtenido a partir de superposición de aportaciones individuales, no fruto de una campaña estructurada dentro del sector, por lo que se evidencia una falta de homogeneidad en los datos y en el tratamiento. 
TABla 1.- INVENTARIOS DE LOS CEMENTOS UTILIZADOS (SiMAPRO E INTRON)

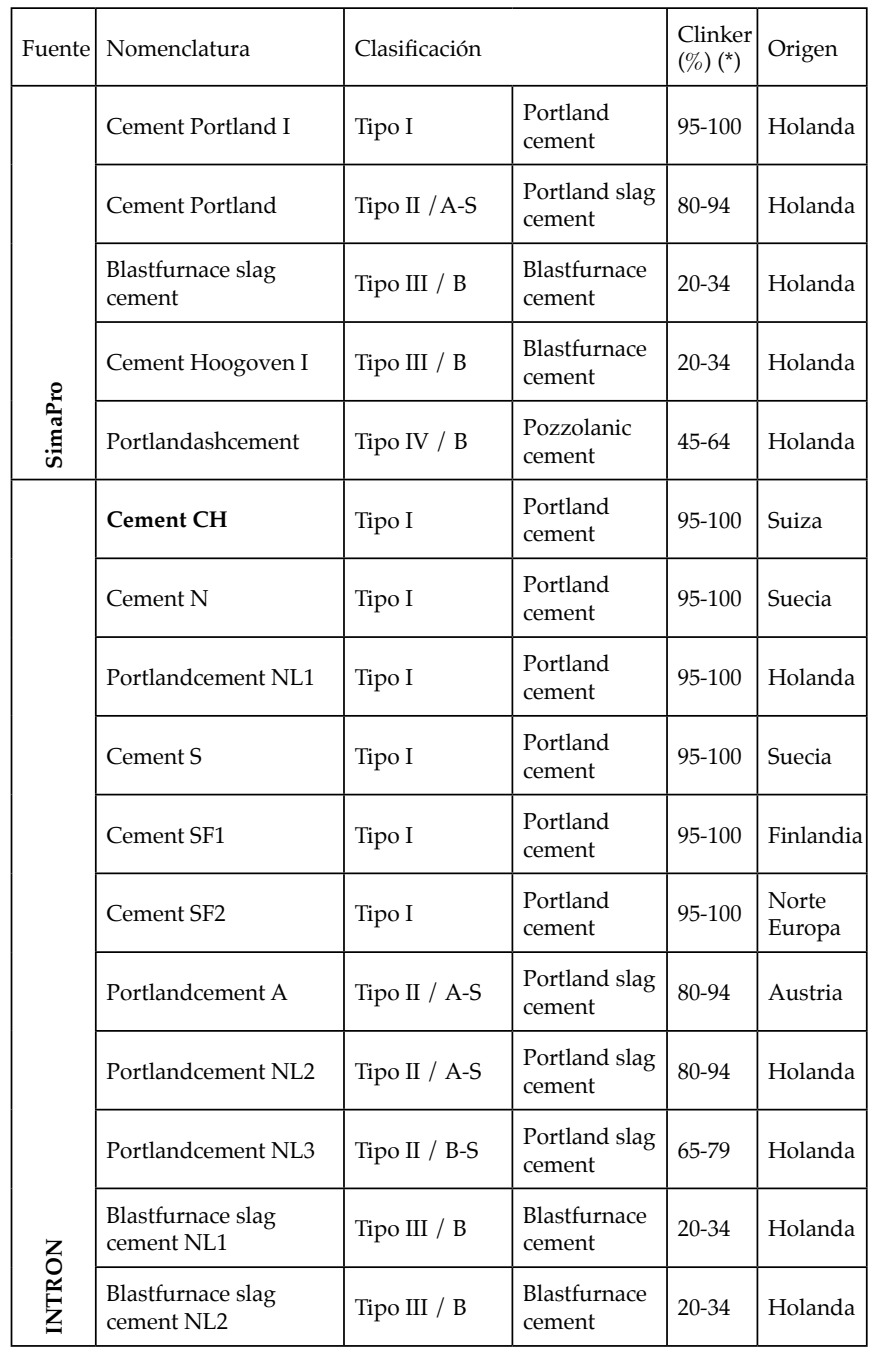

En la citada tabla 1 puede verse que, mientras los inventarios del SimaPro toman principalmente un cemento de cada tipo, en el inventario de INTRON existe una muestra más amplia que permite comparar cementos del mismo tipo, si bien de distinto origen. Con relación al origen hay que señalar, que algunos de los inventarios corresponden a los datos de un cemento y una planta (por ejemplo el Cement $\mathrm{N}$ de la planta de Skövde en Suecia), mientras que otros hacen referencia a un país (por ejemplo, el Portlandcement A de Austria).

Por otro lado, hay que señalar que las salidas de los inventarios citados son distintas entre sí, tal como muestra [1]. Así el inventario de INTRON da una representación gráfica de las etapas que se incluyen en el sistema correspondiente a $1 \mathrm{~kg}$ de cemento, lo cual facilita el entendimiento de cuáles son los límites del sistema, mientras que en el inventario de SimaPro, esa representación gráfica de los límites se ha de construir a partir de la información del texto, lo cual puede aumentar la probabilidad de error al interpretarlo. Respecto al tipo de cemento cabe destacar que prácticamente se dispone de, al menos, un caso de cada uno de los cementos tipo más usuales. Ahora bien, hay que señalar que en la clasificación realizada para situarlos de acuerdo a la EN-197-1 (2001) se han encontrado ciertas dificultades.

Otro aspecto a reseñar es que no siempre se conocía el contenido de clinker de forma directa, en cuyo caso, se determinaba restando de la masa de cemento el contenido de adiciones más el yeso. De lo anterior se concluye ciertas dificultades en la estructuración de la información aportada en los inventarios. Por último, en cuanto a la distri- bución geográfica, hay que señalar que los países de los que proceden los inventarios vienen a representar menos del 15\% de la producción de cemento de la Unión Europea, en el año de 1997. Ello puede reflejar, por un lado, que ante una mayor sensibilización social, los gobiernos dictan normas más estrictas o bien los colectivos empresariales están más en línea con la preocupación social. Por otro lado, esta situación puede ser reflejo de una falta de transparencia en los datos disponibles, los cuales (en el caso de disponerse) sólo son de uso interno de las fábricas o, como máximo, del sector.

\section{ANÁLISIS DE LOS DATOS DE ENTRADA}

\subsection{El consumo de materias primas}

El sistema elegido se puede desglosar en otros subsistemas. La estructuración que se refleja en los inventarios utilizados es la de un único sistema (INTRON) o bien, según SimaPro, la división en los dos subsistemas siguientes: Producción del clinker + Producción del cemento. En la tabla 2 se presenta un resumen de los datos del consumo de materiales utilizados en la fabricación de $1 \mathrm{~kg}$ de cemento, tal y como se reflejan en las fichas de cada inventario. En la citada tabla puede observarse que los inventarios de INTRON no señalan la cantidad de clinker (de acuerdo con el planteamiento conjunto realizado). Ahora bien, este planteamiento no es el seguido en los inventarios de SimaPro (tratamiento separativo), en los cuales el yeso se suma a partir de la unidad de los otros componentes (excepto en el Cement Portland I).

TABLA 2. CONSUMO DE MATERIAS PRIMAS (EN KG) PARA 1A PRODUCCIÓN DE 1 KG DE CEMENTO

\begin{tabular}{|c|c|c|c|c|c|c|c|c|c|c|c|c|}
\hline \multirow{3}{*}{ Tipo } & \multirow{3}{*}{$\begin{array}{l}\text { Nomenclatura } \\
\text { Original }\end{array}$} & \multicolumn{11}{|c|}{ Consumo de materias primas (en $\mathrm{kg}$ por $\mathrm{kg}$ de cemento) } \\
\hline & & \multicolumn{7}{|c|}{ Producción del clinker } & \multicolumn{4}{|c|}{ Producción del cemento } \\
\hline & & 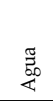 & Uี & 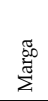 & 愛 & 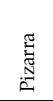 & 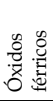 & $\stackrel{\mathscr{I}}{0}$ & 崩 & 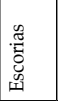 & 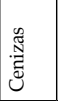 & 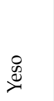 \\
\hline \multirow{5}{*}{ 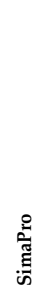 } & Cement Portland I & -- & -- & 1,600 & - & -- & -- & 0.270 & 0,94 & - & - & 0,060 \\
\hline & Cement Portland & -- & -- & 1,610 & 0,057 & 0,047 & 0,019 & -- & 0,95 & 0,109 & 0,09 & 0,050 \\
\hline & Cement Hoogoven I & - & -- & 0,510 & - & -- & -- & 0,066 & 0,30 & 0,640 & - & 0,060 \\
\hline & $\begin{array}{l}\text { Blastfurnace slag } \\
\text { cement }\end{array}$ & -- & -- & 0,425 & 0,015 & 0,012 & 0,005 & -- & 0,25 & 0,729 & 0,024 & 0,050 \\
\hline & Portlandashcement & -- & -- & 1,190 & 0,042 & 0,035 & 0,014 & - & 0,70 & 0,081 & 0,317 & 0,050 \\
\hline \multirow{11}{*}{$\begin{array}{l}Z \\
\text { Z } \\
\stackrel{\Xi}{\mid} \\
z\end{array}$} & Cement $\mathrm{CH}$ & -- & 1,15 & 0,346 & -- & -- & -- & $\mid--$ & -- & |- & |- & 0,030 \\
\hline & Cement N & -- & 1,64 & -- & -- & -- & -- & - & -- & - & - & 0,050 \\
\hline & $\begin{array}{l}\text { Portlandcement } \\
\text { NL1 }\end{array}$ & 1,410 & 1,60 & -- & -- & -- & -- & -- & -- & -- & -- & 0,060 \\
\hline & Cement $\mathrm{S}^{*}$ & -- & 1,36 & -- & -- & 0,046 & 0,009 & -- & -- & - & - & 0,046 \\
\hline & Cement SF1 & -- & 1,20 & -- & -- & -- & -- & $\mid--$ & -- & |- & |- & -- \\
\hline & Cement SF2 & -- & 1,55 & -- & -- & -- & -- & -- & -- & - & - & -- \\
\hline & Portlandcement $\mathrm{A}^{*}$ & 0,190 & 1,20 & -- & - & -- & -- & 0,017 & -- & 0,122 & 0,027 & 0,064 \\
\hline & $\begin{array}{l}\text { Portlandcement } \\
\text { NL2 }\end{array}$ & 1,071 & -- & 1,045 & 0,028 & 0,066 & 0,019 & 0,047 & -- & 0,095 & 0,076 & 0,050 \\
\hline & $\begin{array}{l}\text { Portlandcement } \\
\text { NL3 }\end{array}$ & 1,325 & -- & 1,316 & 0,056 & 0,047 & 0,014 & -- & -- & 0,108 & 0,089 & 0,060 \\
\hline & $\begin{array}{l}\text { Blastfurnace slag } \\
\text { NL1 }\end{array}$ & 0,532 & -- & 0,287 & 0,007 & 0,017 & 0,005 & 0,025 & -- & 0,700 & 0,020 & 0,050 \\
\hline & $\begin{array}{l}\text { Blastfurnace slag } \\
\text { NL2 }\end{array}$ & 0,423 & -- & 0,420 & 0,018 & 0,015 & 0,004 & -- & -- & 0,675 & 0,285 & 0,060 \\
\hline
\end{tabular}


Además, en el caso del Portland Cement (II/A-S) la suma de los componentes es superior a la unidad, lo cual debe reflejar la existencia de algún error (al cual no se ha tenido acceso). Así pues, una primera conclusión de estos datos es que los mismos vienen influenciados por la fuente de procedencia. Además parece que existen errores numéricos en algunos de ellos. En la citada tabla 2 puede verse, aparte de la lógica dispersión correspondiente al tipo de cemento, la existencia de límites diferentes del sistema. Así algunos incorporan los explosivos utilizados en las canteras en la extracción de las rocas (Portlandcement A y Cement S), mientras que los otros no inciden en ese término. Asimismo, puede observarse esa dispersión en la inclusión o no del consumo de agua utilizada en el proceso. Un aspecto interesante a reseñar es el orden de magnitud del consumo de caliza, el cual se sitúa en el entorno de 1,60 kg de caliza (y/o marga) / kg de cemento (en los cementos tipo I) y lógicamente menor en los otros tipos. Este factor es importante, dado que la emisión de $\mathrm{CO}_{2}$ está influenciada principalmente por la cantidad de caliza y su transformación en la fabricación del clinker.

\subsection{El consumo de energía}

Tal como señala la ISO 14.042:2000, en su apartado 5.3, el estudio de la energía consumida es uno de los parámetros más relevantes para el chequeo de fiabilidad de los inventarios empleados en el ACV de un producto o proceso. Por ello en la tabla 3 se presenta el consumo de energía (en MJ) requerido, en la fabricación de $1 \mathrm{~kg}$ de cemento, para todos los inventarios y tipos de cementos. La energía total utilizada se desglosa en eléctrica y térmica, tal como se desprende de la información aportada por los inventarios utilizados. En la energía eléctrica suelen incluir la utilizada en las instalaciones provenientes de red externa. Por ello, dependiendo del país de origen, esa energía suele representarse por porcentajes de origen de la misma (nuclear, hidroeléctrica, centrales térmicas, solar, eólica, mareas, otras), en lo que se conoce como matriz energética. Las emisiones en la obtención de estas energías no tienen por qué estar en el entorno de la fábrica de cemento, sino que usualmente estarán lejos de la misma.

La energía térmica corresponde principalmente a la utilizada en el horno de fabricación del clinker y suele utilizar recursos fósiles no renovables (fuel, carbón, etc). En la actualidad, la obtención de los mismos suele ser lejana a la fábrica (por ejemplo, carbón de Australia o Sudáfrica) si bien las emisiones de la fabricación del clinker repercuten en un ámbito local, regional y global.En la citada tabla puede observarse que desde el punto de vista energético (electricidad + energía térmica), el mayor consumo corresponde a la producción del clinker alcanzando casi el $90 \%$ del total de la energía consumida en el sistema. Consecuentemente, los cementos que utilizan menores cantidades de clinker consumen menos energía como era de esperar y es bien conocido. La distribución del consumo energético entre electricidad y energía térmica es aleatoria, sin tener una solución única, parece que depende del tipo de fábrica.

Por otro lado, para el conjunto de los cementos tipo I el valor medio de la energía consumida por kg de cemento es de 4,31 MJ, lo cual está en orden de magnitud con los valores expresados por INTRON, que los sitúa, en 1995, sobre 3,7 MJ / kg de clinker, valor éste al que habría que sumar la energía correspondiente en la producción del cemento, que tal como se ha dicho para un cemento tipo I está entre el 10\% y el 15\% de la anterior, lo que conduciría al valor medio antes citado $(4,31 \mathrm{MJ} / \mathrm{kg})$.
TABLA 3.- CONSUMO DE ENERGÍA (EN MJ) EN LA PRODUCCIÓN DE 1 KG DE CEMENTO (ELECTRICIDAD Y TÉRMICA)

\begin{tabular}{|c|c|c|c|c|c|c|}
\hline \multirow{2}{*}{ Tipo } & \multirow{2}{*}{ Nomenclatura } & \multicolumn{2}{|l|}{ Clinker } & \multicolumn{2}{|l|}{ Cemento } & \multirow{2}{*}{$\begin{array}{l}\text { Suma } \\
(100 \%)\end{array}$} \\
\hline & & $\begin{array}{c}\text { Térmica } \\
(\%)\end{array}$ & $\begin{array}{c}\text { Electricidad } \\
(\%)\end{array}$ & $\begin{array}{c}\text { Térmica } \\
(\%)\end{array}$ & $\begin{array}{c}\text { Electricidad } \\
(\%)\end{array}$ & \\
\hline \multirow{7}{*}{ I } & Cement Portland I & $3,38(74)$ & $0,196(4)$ & $0,846(19)$ & $0,122(3)$ & 4,544 \\
\hline & Cement $\mathrm{CH}$ & & & 2,980 (84) & 0,557 (16) & 3,537 \\
\hline & Cement N & & & $3,850(87)$ & 0,557 (13) & 4,407 \\
\hline & \begin{tabular}{|l|} 
Portlandcement \\
NL1
\end{tabular} & $3,38(92)$ & $0,196(5)$ & & $0,122(3)$ & 3,698 \\
\hline & Cement S & & & $4,070(90)$ & $0,470(10)$ & 4,540 \\
\hline & Cement SF1 & & & $4,900(92)$ & $0,450(8)$ & 5,350 \\
\hline & Cement SF2 & $3,77(91)$ & 0,136 (3) & 0,029 (1) & $0,189(5)$ & 4,124 \\
\hline \multirow{4}{*}{ II } & Cement Portland & $3,38(92)$ & $0,134(4)$ & $0,032(0)$ & $0,146(4)$ & 3,692 \\
\hline & Portlandcement A & & & $2,810(88)$ & $0,382(12)$ & 3,192 \\
\hline & \begin{tabular}{|l|} 
Portlandcement \\
NL2 \\
\end{tabular} & & & $2,640(96)$ & $0,116(4)$ & 2,756 \\
\hline & \begin{tabular}{|l|} 
Portlandcement \\
NL3
\end{tabular} & & & $3,380(91)$ & $0,318(9)$ & 3,698 \\
\hline \multirow{4}{*}{ III } & \begin{tabular}{|l} 
Cement Hoogoven \\
I
\end{tabular} & $1,08(43)$ & $0,063(2)$ & $1,080(43)$ & $0,292(12)$ & 2,515 \\
\hline & \begin{tabular}{|l}
$\begin{array}{l}\text { Blastfurnace slag } \\
\text { cement }\end{array}$ \\
\end{tabular} & $0,88(55)$ & $0,035(2)$ & $0,120(8)$ & $0,551(35)$ & 1,587 \\
\hline & \begin{tabular}{|l|} 
Blastfurnace slag \\
NL1 \\
\end{tabular} & & & $0,680(70)$ & $0,287(30)$ & 0,967 \\
\hline & \begin{tabular}{|l} 
Blastfurnace slag \\
NL2
\end{tabular} & & & $1,080(75)$ & $0,354(25)$ & 1,434 \\
\hline IV & $\begin{array}{l}\text { Portland ash } \\
\text { cement }\end{array}$ & $2,49(89)$ & 0,099 (3) & $0,042(1)$ & $0,194(7)$ & 2,825 \\
\hline
\end{tabular}

\section{LAS SALIDAS DEL SISTEMA}

Para analizar la fiabilidad de un inventario, un parámetro fundamental es el estudio de las emisiones más significativas del sistema. Entre ellas se encuentran el $\mathrm{CO}_{2}$, por su importante influencia en el efecto invernadero y el carácter global de la misma, el $\mathrm{NO}_{x}$ y el $\mathrm{SO}_{2}$ por su influencia en la acidificación y eutrofización (el primero) y el carácter regional de ambos y, el polvo por la importante repercusión visual y directa con un efecto local sobre imagen y salud del entorno y sus habitantes. Para garantizar la consistencia de los resultados se ha incluido en el sistema de producción de $1 \mathrm{~kg}$ de cemento, las emisiones de los parámetros elegidos $\left(\mathrm{CO}_{2^{\prime}} \mathrm{NO}_{x^{\prime}} \mathrm{SO}_{2^{\prime}}\right.$ polvo) por cada $\mathrm{MJ}$ de energía eléctrica utilizado, lo que requiere conocer la respectiva matriz energética (procedencia de la energía) del país de origen del cemento que explícitamente indican cada inventario.

Dadas las limitaciones de espacio existentes del artículo, como ejemplo, en lo que sigue se toma uno sólo de los factores analizados, las emisiones de $\mathrm{CO}_{2}$. Un mayor detalle del estudio de este factor y del resto de factores, puede verse en [1]. Las emisiones de $\mathrm{CO}_{2}$ en el sistema elegido de $1 \mathrm{~kg}$ de cemento, se sitúa principalmente en las siguientes etapas: Cantera de extracción materiales (1\%), molienda y prehomogenización $(4 \%)$, horno del clinker $(87 \%)$, molienda del cemento (5\%), distribución interna planta (3\%).

Aparte de los datos registrados en cada inventario se ha hecho una estimación numérica de los mismos, para cada uno de los cementos, a partir del modelo propuesto por la IPCC [4]. Con ello se pretendía tener una herramienta de análisis en paralelo, sobre la fiabilidad de los datos registrados en cada inventario. En la tabla 4 se muestran las emisiones de $\mathrm{CO}_{2}$ de los registros de cada inventario y las obtenidas a partir del modelo citado. 
TABLA 4.- EMISIONES DE CO (EN GRAMOS) POR KG DE CEMENTO, EN CADA ETAPA SEGÚN CÁLCULO Y LAS TOTALES REGISTRADAS

\begin{tabular}{|c|c|c|c|c|c|c|}
\hline \multirow{3}{*}{ Tipo } & \multirow{3}{*}{$\begin{array}{l}\text { Nomenclatura } \\
\text { Original }\end{array}$} & \multicolumn{5}{|c|}{ Emisiones de $\mathrm{CO}_{2}$ (gramos) } \\
\hline & & \multicolumn{4}{|c|}{ Cálculo teórico según modelo IPCC } & \multirow{2}{*}{$\begin{array}{c}\text { Según } \\
\text { inventarios }\end{array}$} \\
\hline & & $\begin{array}{c}\text { Des- } \\
\text { carbonatación }\end{array}$ & \begin{tabular}{|c|} 
Combustibles \\
fósiles
\end{tabular} & Electricidad & Suma & \\
\hline & & (A) & (B) & (C) & $(\mathrm{A}+\mathrm{B}+\mathrm{C})$ & (D) \\
\hline \multirow{7}{*}{ I } & Cement Portland I & 476,67 & 309,07 & 55,97 & 841,71 & 355,00 \\
\hline & Cement $\mathrm{CH}$ & 491,89 & 277,10 & 74,08 & 843,06 & 810,00 \\
\hline & Cement $\mathrm{N}$ & 481,75 & 352,89 & 14,38 & 849,01 & 813,00 \\
\hline & Portlandcement NL1 & 476,67 & 261,61 & 66,14 & 804,43 & 853,00 \\
\hline & Cement S & 481,75 & 368,06 & 4,439 & 854,24 & 805,00 \\
\hline & Cement SF1 & 481,75 & 379,26 & 50,40 & 911,41 & 780,00 \\
\hline & Cement SF2 & 481,75 & 294,12 & 15,86 & 791,73 & 812,70 \\
\hline \multirow{4}{*}{ II } & Cement Portland & 481,75 & 263,93 & 34,16 & 779,84 & 918,30 \\
\hline & Portlandcement A & 400,61 & 244,09 & 30,71 & 675,41 & 586,00 \\
\hline & Portlandcement NL2 & 395,54 & 237,52 & 24,13 & 657,19 & 807,00 \\
\hline & Portlandcement NL3 & 375,25 & 261,61 & 66,14 & 703,01 & 289,00 \\
\hline \multirow{4}{*}{ III } & Cement Hoogoven I & 152,13 & 144,18 & 62,48 & 358,79 & 221,70 \\
\hline & $\begin{array}{l}\text { Blastfurnace slag } \\
\text { cement }\end{array}$ & 126,78 & 77,477 & 71,49 & 275,74 & 334,00 \\
\hline & Blastfurnace slag NL1 & 116,63 & 61,779 & 59,70 & 238,11 & 212,00 \\
\hline & Blastfurnace slag NL2 & 121,70 & 83,592 & 73,63 & 278,93 & 134,00 \\
\hline IV & Portlandashcement & 354,97 & 195,82 & 35,75 & 586,54 & 692,90 \\
\hline
\end{tabular}

Las correspondientes al modelo se han desglosado por cada una de las etapas antes citadas, agrupadas como sigue:

- Resultado de la reacción química en el horno de obtención del clinker (A)

- Combustible utilizado en la obtención del clinker (B)

- Energía consumida en todo el proceso (tanto clinker como cemento) (C)

En primer lugar puede observarse la buena concordancia entre los valores registrados y los estimados. En la misma puede apreciarse que las emisiones de $\mathrm{CO}_{2}$ corresponden, principalmente (más del 90 $\%$ en los cementos tipo I), a las emisiones derivadas de las reacciones químicas que se producen en el horno de fabricación del clinker y de los combustibles utilizados en el mismo. Las menores emisiones que se producen en los cementos a medida que aumentan las adiciones (cementos tipo III y IV), son consecuencia de que éstas generan menos $\mathrm{CO}_{2}$. Además, en ellas no se ha tenido en cuenta el efecto favorable de reutilizar un residuo industrial. La explicación pormenorizada de algunos de los resultados atípicos puede encontrase en [1].

Por otro lado, las emisiones de $\mathrm{CO}_{2}$ correspondientes a la energía eléctrica utilizada representan un porcentaje pequeño, que en el caso de los cementos tipo I no llega al $10 \%$, aumentando este porcentaje a medida que el valor absoluto disminuye. Ello es consecuencia de que los valores procedentes de la energía corresponden, principalmente, a los equipos de manipulación utilizados

En cuanto a la experiencia española, recogida por OFICEMEN [5], en el año 1996, sobre este punto se señala que el $59 \%$ de las emisiones de $\mathrm{CO}_{2}$ corresponden a las reacciones químicas producidas en la fabricación del clinker, el $35 \%$ es consecuencia del consumo de combustibles en dicha etapa y el $6 \%$ de estas emisiones corresponde al consumo de energía en todas las etapas. En este punto cabe recordar la matización de que el clinker puede ser producido en un lugar diferente del cemento, cara a tenerlo presente en los efectos locales o regionales.

Por su parte, la experiencia de Dinamarca se recogen en [6], señalando que se producen unas emisiones de $\mathrm{CO}_{2}$ entre 0,1 y 0,2 toneladas por cada tonelada de hormigón producido, que representa un orden de magnitud similar a los anteriormente expresados. Sin embargo, respecto a la energía total consumida en todo el proceso, los citados autores reflejan valores entre 3 y $4 \mathrm{MJ} / \mathrm{kg}$, que representan valores algo inferiores a los anteriormente citados en los distintos inventarios.

\section{Conclusiones}

Del análisis de los datos de entradas y salidas (emisiones de $\mathrm{CO}_{2}$ ) del sistema de producción de $1 \mathrm{~kg}$ de cemento de los inventarios analizados se desprende las siguientes conclusiones principales:

- Los límites de los sistemas de los diferentes cementos existentes en los inventarios no son homogéneos, lo cual dificulta, lógicamente, los análisis posteriores restando fiabilidad a trabajos comparativos que puedan realizarse con soluciones de diversos materiales (acero, hormigón, etc) y, consecuentemente, se impone la necesidad de ahondar en esta dirección y avanzar en la definición de un modelo nacional de inventario, definiendo bien los subsistemas que se integran en el proceso.

- Existen modelos teóricos que, con base a resultados medios, pueden acotar las emisiones y servir como referencia a los valores medidos y, en algunos casos, ser una alternativa razonable de minimización de campañas de medidas de campo.

- Las emisiones de $\mathrm{CO}_{2}$ se producen principalmente en la fabricación del clinker, tanto por las reacciones químicas que se originan, como por la quema de los combustibles fósiles. Las emisiones correspondientes a la energía eléctrica utilizada en todo el proceso de producción son del orden del $10 \%$ del total, siendo el consumo de energía necesaria para la producción de 1 $\mathrm{kg}$ de cemento del orden de $4 \mathrm{MJ} / \mathrm{kg}$,

\section{AGRADECIMIENTOS}

Los autores expresan su agradecimiento al Instituto Español del Cemento y sus Aplicaciones (IECA) y a la CICYT por los proyectos concedidos y financiados en este ámbito.

\section{BIBLIOGRAFÍA}

1. A. Cardim. "Análisis del ciclo de vida de productos derivados del cemento. Aportaciones al análisis de los inventarios del ciclo de vida del cemento". Tesis doctoral. UPC. Barcelona. Abril 2001. 297 páginas

2. INTRON "An overview of available LCI Data: Cement, concrete and other building material". Institute for Material and Environmental Research B.V. INTRON Report $\mathrm{n}^{\circ}$ 97079. Jun/97-344 pp

3. PRé-Consultants, B.V. "SimaPro - The software tool to analyse and develop environmentally sound products - Single User - Database Manual". 65 páginas. 1997

4. IPCC - OCDE - AIE (1996) Hougton, JT et al (1996) - Greenhouse Gas Inventory - Revised 1996 IPCC Guidelines for National Greenhouse Gas Inventories - Reference Manual - Volume 3 - IPCC/OECD/IEA - Grupo Intergubernamental de Expertos sobre Cambios Climáticos.

5. OFICEMEN “Principales datos del sector cementero. Agrupación de Fabricantes de cemento de España- [http:/ www.oficemen.com].

6. M. Glavind and C. Munch-Petersen. "Green concrete in Denmark". Structural Concrete. 2000, 1, No.1 March, pp 19-25

Recibido: 1.2 .03

Aceptado: 30.11 .03 